\title{
Lettre à la rédaction : Dépistage du cannabis dans les urines : attention à l'acide niflumique
}

\author{
Letter to the editor: \\ Immunoassay for cannabis in urine: \\ beware niflumic acid
}

Hervé DELACOUR*, Aurélie SERVONNET, Véronique GARDET

Fédération de Biologie Clinique, Hôpital d'Instruction des Armées Robert Picqué, 331, route de Toulouse 33140 VILLENAVE D'ORNON

*Auteur à qui adresser la correspondance : Hervé DELACOUR, Fédération de Biologie Clinique, Hôpital d'Instruction des Armées Robert Picqué, 331, route de Toulouse - 33140 VILLENAVE D'ORNON Tél : 0556847302 - Fax : 0556847495 - E-mail : h_delacour@yahoo.fr

(Reçu le 19 avril 2005 ; accepté après modifications le 12 août 2005)

\section{Observation}

Mr. T., 54 ans, personnel navigant de l'armée de l'air, effectue une visite médicale d'aptitude. Dans ce cadre, un dépistage de stupéfiant (cannabis, cocaïne et opiacés) est effectué dans les urines. Il se révèle franchement positif pour les dérivés cannabinoïdes avec la trousse Roche sur Cobas Integra ${ }^{\circledR} 400$ (Cannabinoids II, seuil à $20 \mathrm{ng} / \mathrm{mL}$, concentration de l'échantillon estimée à 204 $\mathrm{ng} / \mathrm{mL}$ ) et le test unitaire Dade Behring (Syva ${ }^{\oplus}$ Rapid Test d.a.u. ${ }^{\otimes} \mathrm{THC}$, seuil à $50 \mathrm{ng} / \mathrm{mL}$ ). L'échantillon est envoyé pour confirmation au laboratoire de chimie analytique de l'Institut de Médecine Aérospatiale du Service de Santé des Armées (Brétigny sur Orge). La réalisation d'une chromatographie en phase gazeuse couplée à la spectrométrie de masse (CPG-SM) infirme le résultat. La recherche avec un réactif dédié Dade Behring sur Dimension ${ }^{\circledR}$ RXL (Syva Emit ${ }^{\circledast}$ II, seuil à 50 $\mathrm{ng} / \mathrm{mL}$ ) s'avère également négative. L'enquête pharmacologique menée auprès du patient dévoile uniquement la prise de Nifluril $^{\oplus}$ (acide niflumique) la veille à une posologie non communiquée. Une interférence liée à ce médicament est suspectée et, aucune des notices techniques des réactifs utilisés ne la mentionnant, une procédure de réactovigilance est engagée auprès de l'Afssaps et des deux fournisseurs. Après enquête, ces derniers confirment l'interférence et annoncent une modification prochaine de leur fiche technique. 


\section{Discussion}

\section{Une interférence déjà connue mais à confirmer pour chaque technique.}

L'interférence des anti-inflammatoires non stéroïdiens (AINS), dérivés des acides arylcarboxyliques, a été signalée dès 1989 par Lafargue et coll. (1). Ces auteurs avaient constaté que l'acide tiaprofénique, dérivé de l'acide propionique, était responsable d'absorbances parfois très importantes à $340 \mathrm{~nm}$, gênantes pour apprécier correctement une variation de densité optique et perturbant donc les techniques EMIT. Lafargue et coll. notaient que cette interférence n'est pas une réaction croisée avec l'anticorps, mais un phénomène d'absorbance dans l'ultra-violet, compte tenu de la structure moléculaire des composés.

En 2002, l'interférence de l'acide niflumique $\left(\right.$ Nifluril $\left.^{\circledR}\right)$, AINS de la classe des fénamates, a été rapportée par Forzy et coll. (2). Dans leur article, la prise d'acide niflumique chez un enfant de 4 ans et demi a entraîné un résultat faussement positif avec deux tests immunochromatographiques (Triage 8 Biosite $^{\circledR}$ et DakoRapide ${ }^{\ominus}$ ) alors que l'étude des mêmes prélèvements avec la trousse Abbott sur $\mathrm{Axym}^{\otimes}$ en polarisation de fluorescence s'est révélée négative. Ces auteurs n'ont pu établir si l'interférence provenait du principe actif, d'un de ses métabolites ou de l'excipient (glycérides hémisynthétiques solides). Pour ces auteurs, les différences structurales entre l'acide 11 -nor- $\delta$-9-tétrahydrocannabinol-carboxylique $(\Delta 9 \mathrm{COOH}-\mathrm{THC})$, composé détecté avec les techniques de dépistage urinaire des cannabinoïdes, et l'acide niflumique ou le morniflumate, rendent peu probable l'existence d'une réaction croisée (figure 1). Cependant, un phénomène d'absorption moléculaire, comme pour les dérivés des acides arylcarboxyliques, explique difficilement ces interférences, les tests utilisés reposant sur une méthode immunochromatographique avec des anticorps marqués à l'or colloïdal.

Notre observation objective la sensibilité de deux autres méthodes à la présence de Nifluril $^{\circledR}$ : le test immunochromatographique Syva ${ }^{\otimes}$ Rapid Test d.a.u. ${ }^{\circledast}$ THC et la trousse Roche sur Cobas Integra ${ }^{\circledR} 400$. Les études menées suite à la réactovigilance (surcharge d'urines exemptes de $\Delta 9 \mathrm{COOH}-\mathrm{THC}$ par de l'acide niflumique, à des concentrations de 3 à $10 \mu \mathrm{g} \cdot \mathrm{mL}^{-1}$ ) ont démontré l'origine de l'interférence : l'acide niflumique. Ainsi, pour le test $S_{y v a}{ }^{\circledR}$, un résultat « faux positif » est observé pour une concentration de $5,4 \mu \mathrm{g} \cdot \mathrm{mL}^{-1} \mathrm{~d}^{\prime}$ acide niflumique (communication de la société Dade Behring).

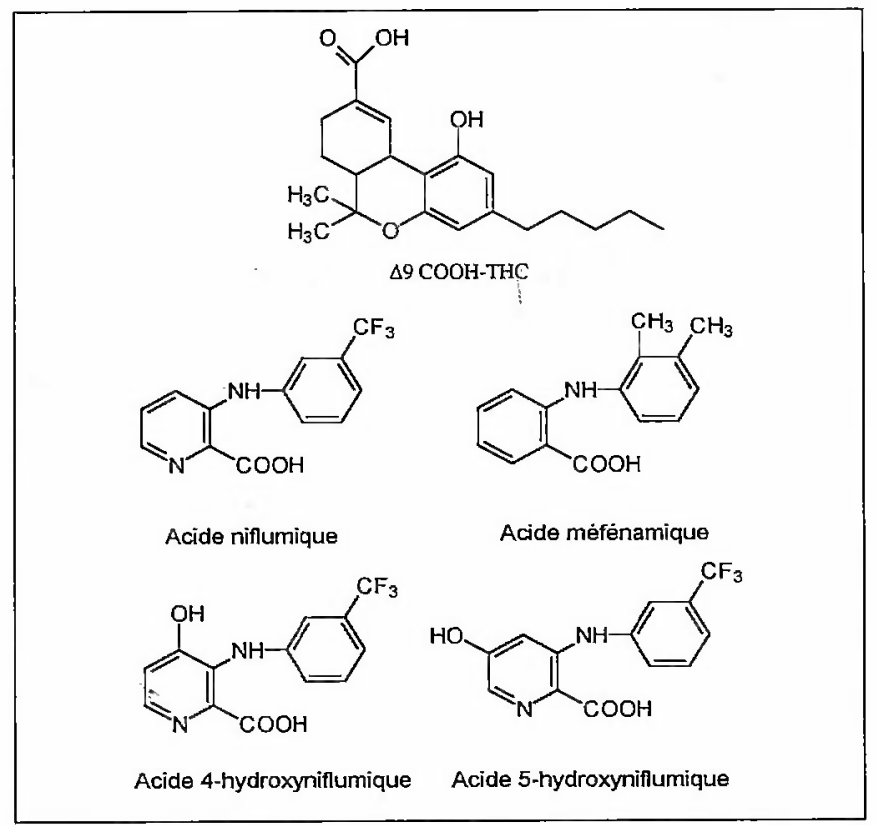

Figure 1 : Fonnules des molécules de $\triangle 9 \mathrm{COOH}-\mathrm{THC}$ d'acide niflumique, d'acide méfénamique et des dérivés métaboliques de l'acide niflumique (acide 4 hydroxyniflumique et acide 5 hydroxynifumique).

Comment expliquer ces faux positifs? Au vue des principes méthodologiques des techniques incriminées (figures 2 et 3 ), un simple phénomène d'absorption moléculaire peut difficilement être mis en cause. Bien qu'écartée lors des observations précédentes, une réaction croisée de l'acide niflumique ou de l'un de ses dérivés métaboliques avec l'anticorps de dosage peut être envisagée. Avec la trousse Roche, une réaction croisée limite l'élaboration du réseau de particules ; l'absorbance du milieu à $659 \mathrm{~nm}$ est diminuée et la concentration en $\Delta 9 \mathrm{COOH}-\mathrm{THC}$ surestimée. Dans le cas du test immunochromatographique Syva, la formation d'un complexe Ac*-acide niflumique empêche la liaison de l'Ac* avec le conjugué de drogue immobilisé sur la membrane. L'absence de bande colorée au niveau du sigle THC est interprétée positive (figure 4). En outre, il est intéressant de souligner les différences observées d'une trousse à l'autre chez un même fabricant. La $2^{\text {ime }}$ génération de la trousse Roche sur Cobas Intégra $^{\circledast}$ est sensible à la présence d'acide niflumique, à $l^{\prime}$ inverse de la $1^{\text {tre }}$ génération, bien que ces deux techniques soient fondées sur la même méthodologie, la seule différence provenant des anticorps utilisés. Si le test immunochromatographique Syva est perturbé, la technique Syva Emit ${ }^{\circledast}$ II sur Dimension ${ }^{\oplus}$ RXL est, quant-à-elle, insensible au Nifluril ${ }^{\circledast}$... 


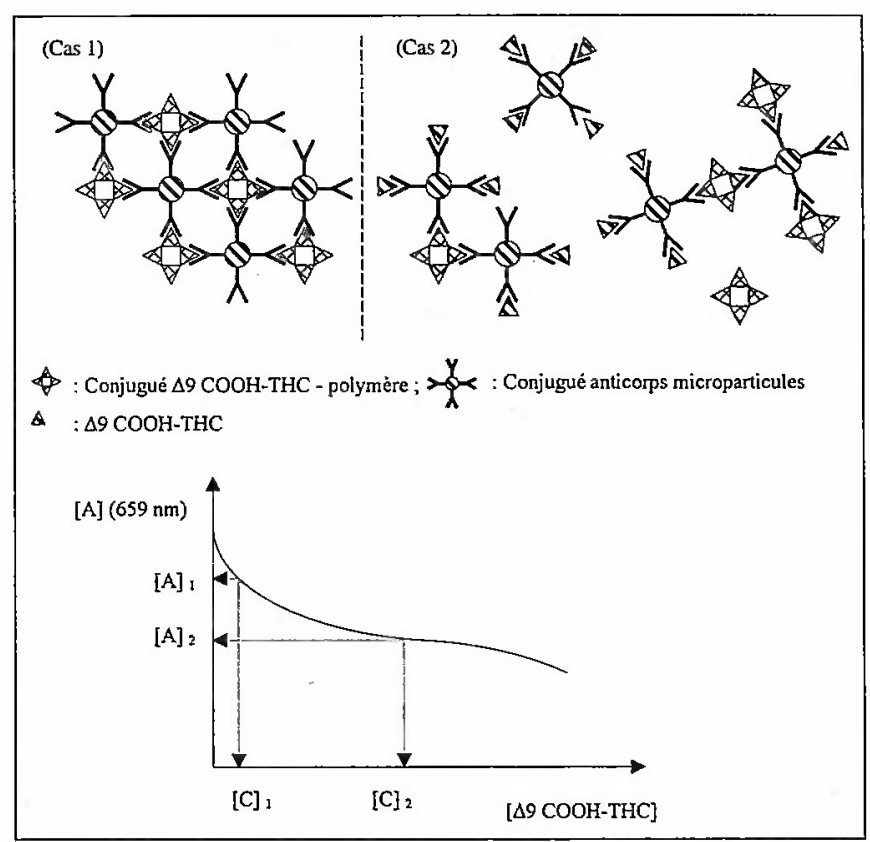

Figure 2 : Principe de la technique Roche sur Cobas Integra ${ }^{\otimes} 400$ (Cannabinoids II). En l'absence de $\triangle 9 \mathrm{COOH}$ THC dans les urines (cas 1), les conjugués drogue-polymère se fixent sur les anticorps liés aux microparticules, aboutissant à la formation de réseaux. Si l'échantillon urinaire contient des dérivés cannabinoïdes (cas 2), le $\triangle 9 \mathrm{COOH}$ THC entre en compétition avec les conjugués drogue-polymère vis à vis des anticorps; la formation du réseau de particules est limitée. La concentration en $\triangle 9$ COOH-THC est inversement proportionnelle à l'absorbance du milieu, mesurée à $659 \mathrm{~nm}(3)$.

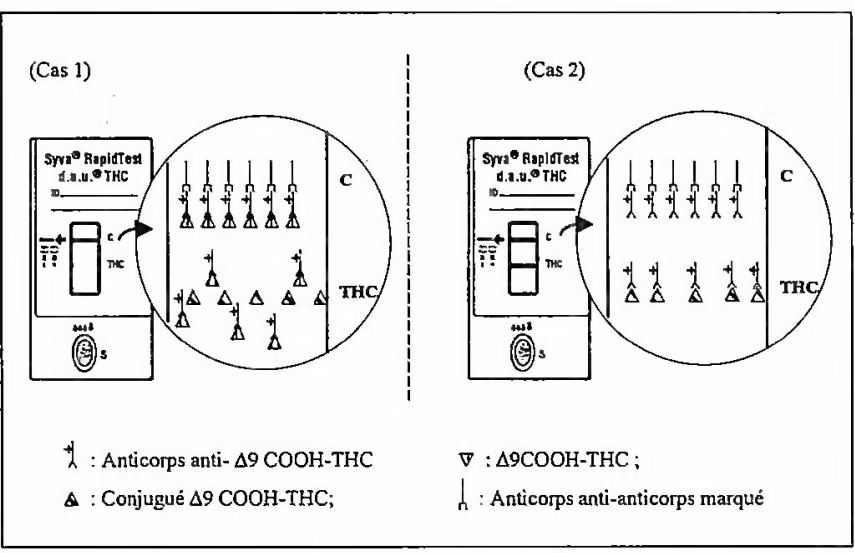

Figure 3 : Principe de la technique Syva $a^{\circledast}$ Rapid Test d.a. $u^{\circledast}$ THC. Un échantillon de l'urine à analyser $(110 \mu \mathrm{L})$ est déposé dans le puit échantillon " $S$ " du dispositif. Par migration, l'échantillon atteint une zone où sont déposés des anticorps anti- $49 \mathrm{COOH}-\mathrm{THC}$ marqués à l'or colloüdal (AC*). En présence de dérivés cannabinoüdes, des complexes $\triangle 9 \mathrm{COOH}-\mathrm{THC}-\mathrm{Ac}^{*}$ se forment. A un seuil de $50 \mathrm{ng} \cdot \mathrm{mL}^{-1}$ de $\triangle 9 \mathrm{COOH}-\mathrm{THC}$, les anticorps marqués sont saturés et ne peuvent se lier aux conjugués de drogues immobilisés sur la membrane. Aucune bande colorée n'apparaît dans la fenêtre résultat au niveau du sigle THC (cas I). A l'inverse, en l'absence de dérivés cannabinoïdes, des complexes Ac* - conjugués de drogue sont formés. Une bande colorée au niveau du sigle THC signe un résultat négatif. Dans les deux cas, une bande contrôle $(C)$, à l'aide d'un anticorps anti-anticorps marqué, pernet de valider le test.

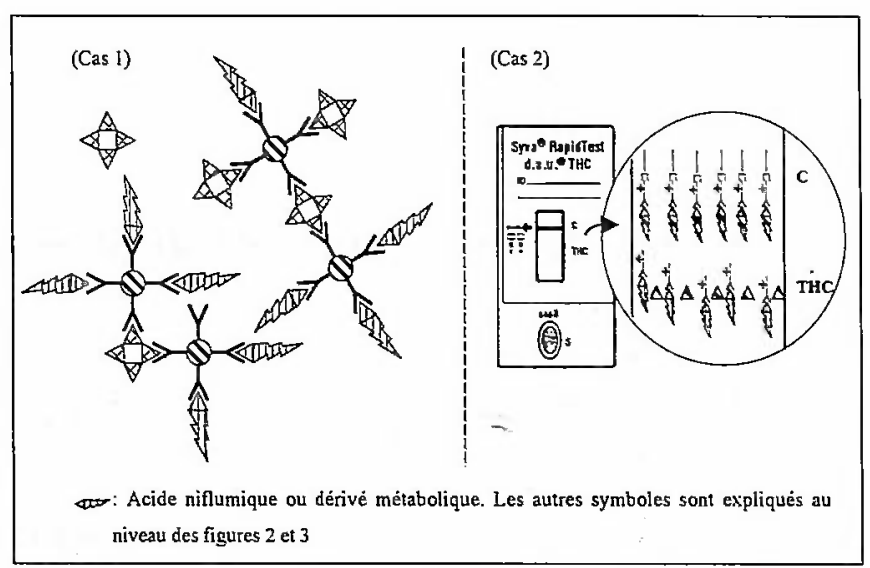

Figure 4: Mécanismes proposés à l'origine des interférences. L'existence d'une réaction croisée entre l'acide niflumique ou l'un de ses dérivés métaboliques et l'anticorps de dosage peut expliquer l'apparition des faux positifs avec les deux techniques. Avec la trousse Roche, la réaction croisée limite l'élaboration du réseau de particules; l'absorbance du milieu à $659 \mathrm{~nm}$ est diminuée et la concentration en $\triangle 9 \mathrm{COOH}$-THC surestimée (cas I). Dans le cas du test immunochromatographique Syva, la formation d'un complexe Ac*-acide niflumique empêche la liaison de l'Ac* avec le conjugué de drogue immobilisé sur la membrane. L'absence de bande colorée au niveau du sigle THC est interprétée positive.

\section{D'autres interférences sont-elles à sus- pecter ?}

Young évoque l'ibuprofène, dérivés des acides arylcarboxyliques (3). Cependant, selon les notices techniques, cette molécule ne croise ni avec le test Triage $8^{\circledR}(4)$, ni avec la technique Syva ${ }^{\circledR}(5)$, ni avec la trousse Roche sur Cobas Integra ${ }^{\circledR} 400$ (6), ou la trousse Dade Behring Dimension ${ }^{\otimes}$ RXL (7). L'acide méfénamique, autre AINS de structure proche de l'acide niflumique, pourrait entraîner également des faux positifs, si une réaction croisée est à l'origine de l'interférence du Nifluril $^{\circledR}$ (figure 1).

A notre connaissance, aucune étude exhaustive de la sensibilité des techniques de dépistage urinaire des cannabinoïdes vis à vis de l'acide niflumique ou d'autres AINS n'a été réalisée. Chaque laboratoire peut réaliser des ajouts dosés d'acide niflumique ou de toute autre substance susceptible d'entraîner une interférence dans des urines exemptes de dérivés cannabinoïdes et déterminer ainsi la spécificité analytique de leur technique. Cependant, cette méthode ne fournirait qu'une réponse partielle, une voire deux techniques étant testées par le laboratoire vis-à-vis d'un éventail limité des molécules, de par le coût de l'étude et la difficulté d'approvisionnement en matière première. Le recours à une campagne de contrôle externe de qualité permettrait de pallier ces limites en testant la majorité des techniques commercialisées. Les informations 
recueillies constitueraient des critères importants lors du choix d'un test de dépistage, du fait de l'usage très répandu de ces spécialités pharmaceutiques.

\section{Quelle conduite tenir en cas de suspicion d'interférence?}

Toutes les notices indiquent qu'un résultat positif avec une technique immunologique doit être confirmé par une méthode de référence, telle que la CPG-SM, pour avoir une valeur médico-légale. Cependant, les conséquences d'un dépistage positif, même à titre temporaire, peuvent être préjudiciables.

Dans notre cas, dans l'attente des résultats de la technique de référence, l'étude d'un second échantillon urinaire environ 48 heures après arrêt du Nifluril ${ }^{\circledast}$ aurait permis d'infirmer ou de confirmer rapidement la suspicion d'interférence. Les médicaments persistant dans l'organisme cinq demi-vie après la dernière prise, l'acide niflumique est totalement éliminé de l'organisme 30 heures après l'arrêt du traitement et est, par conséquent, absent des urines au bout de 48 heures (8). A l'inverse, l'élimination des cannabinoïdes est lente, du fait de l'existence d'un cycle entéro-hépatique et d'une réabsorption rénale. La demi-vie d'élimination du tétrahydrocannabinol est d'environ 8 jours et certains auteurs ont retrouvé ses métabolites dans les urines de fumeurs réguliers 72 jours après la dernière prise (9). L'obtention d'un résultat négatif sur un second prélèvement est en faveur d'une interférence, et ce d'autant plus que le premier résultat est fortement positif. Ainsi, dans le cas rapporté par Forzy et coll. (2), une seconde recherche au bout de 72 heures s'est révélée négative. Le même raisonnement peut être mené pour d'autres principes actifs. Bien entendu, cette démarche ne se substitue en aucun cas à la confirmation par une technique de référence sur le premier échantillon, seule démarche permettant de conclure à une inaptitude professionnelle.

Depuis cette première observation, d'autres faux positifs liés à la prise d'acide niflumique ont été observés dans notre laboratoire. Cette interférence illustre une nouvelle fois les limites des techniques immunologiques de dépistage des stupéfiants. Ces limites des techniques de dépistage doivent être rappelées aux prescripteurs pour une bonne interprétation des résultats.

\section{Références}

1. Lafargue P., Chegaray E., Huart B., Pierard C. Conduites toxicophiles et thérapeutique anti-inflammatoire. Toxicorama $1989 ; 1: 36$.

2. Forzy G., Spyckerelle C., Scalber M., Lhermitte M., Dhondt J.-L. Cannabis et nifluril. Ann. Biol. Clin. 2002 ; $60: 745-6$.

3. Young D.S. Effects of drugs on clinical laboratory tests. Washington: AACC Press, $2000: 60: 2-16$.

4. Menassanch S. Cannabis, Nifluril et Triage ${ }^{\circledR}$. Ann. Biol. Clin. $2003 ; 61: 638$.

5. Anonyme. Notice technique Dade Behring Syva ${ }^{\circledR}$ Rapid Test d.a.u. ${ }^{\otimes}$ THC. Version P-5802-D révisée en août 2003.

6. Anonỳme. Notice technique Cobas Integra(r) 400 Cannabinoids II. Version 1 révisée en février 2004.

7. Anonyme. Notice technique Dade Behring Dimension ${ }^{\circledR}$ RXL Syva Emit ${ }^{\otimes}$ II. Version 2001-02.

8. Anonyme. Monographies Nifluril ${ }^{\otimes}$. Edition Vidal 2002 : 1262-3.

9. Ellis G.M., Mann M.A., Judson B.A., Schram N.T., Tashian A. Excretion of cannabinoid metabolites after last use in a group of chronic users. Clin. Pharmacol. Ther. $1985 ; 38: 572-8$. 